\title{
Performance analysis for T.H.E.M.I.S(*) image stabilizer optical system
}

\section{Anisoplanatism limitations \\ (*)Télescope Héliographique pour l'Etude du Magnétisme et des Instabilités de l'atmosphère Solaire}

\author{
G. Molodij and J. Rayrole \\ Observatoire de Paris URA 326, CNRS, 61 Av. de l'Observatoire, 75014 Paris, France
}

Received March 13; accepted July 1, 1997

\begin{abstract}
Numerical simulations of anisoplanatism effects after compensation by the T.H.E.M.I.S. image stabilizer optical system when observing extended sources are presented. The residual wavefront error after correction is computed using the expansion on the Zernike polynomials to analyze the performances of different adaptive optics systems for solar observations. The long exposure optical tranfer functions are derived to simulate the image quality after correction for extended field of view. We demonstrate the capacity of the image stabilizer system for very large field of view observations with medium image quality while the capacity of high order adaptive optics system is suitable for high image quality observations but to the price of a reduction of the field of view.
\end{abstract}

Key words: telescopes — atmospheric effects techniques: miscellaneous — Sun: granulation

\section{Introduction}

The franco-Italian T.H.E.M.I.S. telescope (T.H.E.M.I.S. is a French acronym which stands for "solar heliographic telescope for the study of solar magnetism and atmospheric instabilities") is designed to obtain very precise measurements of the solar magnetic field vector from simultaneous observations of different polarized spectral lines. Image stabilization and high image quality are requirements for the observation of small solar magnetic features like magnetic flux tubes, so that the light passing through the entrance of the spectrograph slit will come from the same solar structure troughout the observation time ( $\simeq 0.4$ second) which is longer than the caracteristic time of evolution of the terrestrial atmospheric tubulence. One of the main optical elements integrated into
T.H.E.M.I.S. optical train is a tiltable mirror which corrects instrument vibrations and steering as well as random wavefront caused by atmospheric turbulence (Rayrole 1992).

Solar physics research requires observations of magnetic features in a range from one tenth of an arcsecond, for flux tubes, to a few arc-minutes, for active regions. Another demand on the image compensation system is that the observer should be able to analyze a large field of view. So, an estimate of image quality after correction using the T.H.E.M.I.S. image stabilizer system is presented: 1) To analyze the compensation degradation by adaptive optics system across large fields of view taking into account the effects of anisoplanatism.

2) To determine the usable limited field of view of the wavefront analysis corresponding to the isoplanatic patch. Anisoplanatism poses a severe problem if the field of view of the correlation tracker is not cut down to approximately the isoplanatic patch. The new tracking method called granulation tracking described in a previous paper (Molodij et al. 1996) is devoted to measure image motions with extended incoherent sources. The granulation tracker, incorporated in the T.H.E.M.I.S. optical arrangement is designed to work on a square field of view of the granulation image which can be adjusted from $2 \times 2$ arcseconds to $12 \times 12$ arcseconds, depending on the Fried parameter $r_{0}$.

The favorable turbulence conditions at the Izaña site in the canary Island $\left(r_{0}>15 \mathrm{~cm}\right.$ for $60 \%$ of the observation times) (Barletti et al. 1973) suggests that image stabilization system is well adapted for improving image resolution of large field of view observations in visible wavelength with the use of a tiltable mirror. This is due to the fact that T.H.E.M.I.S. is a 0.9 meter class telescope $\left(D / r_{0}<\right.$ 6 for $60 \%$ of the observation times). So, atmospheric 
perturbations are esentially caused by the wavefront tilts whose variances represent $90 \%$ of the total variance phase (Noll 1976).

However, it must be keep in mind that any correlation tracker makes wavefront slope measurements in order to correct pure wavefronts tilts. Such problems are analyzed in this paper when observing extended sources.

A new approach to wavefront sensing on extended, complex objects based on the curvature sensing technique while imaging the Sun has been recently proposed by R. Kupke, F. Roddier and D.L. Mickey (Kupke et al. 1994). The goal of this paper is also to investigate and compare the performances of image stabilizer optical system and adaptive optics system which will be able to correct the first aberrated modes as tilt, focusing and astigmatism for the 0.9 meter telescope.

In order to evaluate the limitations due to angular anisoplanatism and slope measurements by correlation trackers, this paper presents a theoretical analysis based on the modal control of the adaptive optics system. Modal control is an helpful tool in adaptive optics in order to manage optimal correction in terms of temporal and angular decorrelation of turbulent wavefront (Rousset 1993; Gendron \& Léna 1994). The analysis uses Mellin transform techniques to evaluate the effects of anisoplanatism upon the performance of adaptive optics system (Chassat 1989; Sasiela 1994; Molodij \& Rousset 1997).

In Sect. 2, the residual wavefront distortions associated are evaluated modally in terms of Zernike polynomials which are chosen for their simple analytical form and because of the correspondance of the low-order Zernike polynomials to physically controlable modes of correction, .i.e, tilt, focusing, astigmatism, etc. The problem of the wavefront tilts correction from wavefront slope measurements is considered in Sect. 3 using the modal expansion on the Zernike polynomials (Primot et al. 1990).

These analysis are directly related to the Zernike coefficient angular correlations between two plane waves (Chassat 1992). Numerical results are presented in Sect. 4 for the modelized turbulence profile $C_{n}^{2}$ resulting from experimental measurements on the Izaña site by Arcetri university (Barletti et al. 1973). In Sect. 5, the optical transfer functions are evaluated for a Zernike expansion following the Wang and Markey approach (Wang \& Markey 1978). The statistics of the Zernike coefficients for an expansion of Kolmogorov turbulence phase distortion have been derived by Noll (Noll 1976) and applied to calculate the optical transfer function $(O T F)$ for a point source (Wang $\&$ Markey 1978). We develop the calculation of $O T F$ to cases when the observing source is extended in order to evaluate the degradation in the field of view of the image due to anisoplanatism after adaptive optics compensation. We compare the performances of image stabilizer optical system and adaptive optics system which will correct aberrations as tilt, focusing and astigmatism on solar granulation images.

\section{Residual wavefront error}

In order to determine the compensation quality in a field of view, the residual phase variance over the telescope aperture is expressed expanding the turbulent wavefront on the Zernike polynomials (or modes). The properties of Zernike polynomials, denoted $Z_{j}$, are well described by Noll (Noll 1976) whose notation is adopted.

The wavefront $\phi(\boldsymbol{r}, \alpha)$ incoming from a determined area on the Sun, located at angular distance $\alpha$ from the on-axis observed direction, is expanded as (piston mode removed):

$\phi(R \boldsymbol{\rho}, \alpha)=\sum_{j=2}^{\infty} a_{j}(\alpha) Z_{j}(\boldsymbol{\rho})$

where $\boldsymbol{\rho}$ is the normalized position vector in the aperture, $R$ the radius of the telescope aperture and the $a_{j}(\alpha)$ the Zernike expansion coefficients given by:

$a_{j}(\alpha)=\int \mathrm{d}^{2} \boldsymbol{\rho} W(\boldsymbol{\rho}) \phi(R \boldsymbol{\rho}, \alpha) Z_{j}(\boldsymbol{\rho})$

introducing the Noll normalisation function:

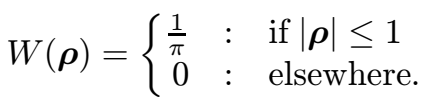

So, the polynomials $Z_{j}(\boldsymbol{\rho})$ normalised on the telescope aperture are defined in polar coordinates $(\rho, \theta)$ by:

$Z_{j}(\rho, \theta)=\sqrt{n+1}\left\{\begin{aligned} R_{n}^{m}(\rho) \sqrt{2} \cos (m \theta) & : \quad j \text { even } m \neq 0 \\ R_{n}^{m}(\rho) \sqrt{2} \sin (m \theta) & : \quad j \text { odd } m \neq 0 \\ R_{n}^{0}(\rho) & : \quad m=0\end{aligned}\right.$

and

$R_{n}^{m}(\rho)=\sum_{s=0}^{\frac{n-m}{2}} \frac{(-1)^{s}(n-s) !}{s !\left[\frac{n+m}{2}-s\right] !\left[\frac{n-m}{2}-s\right] !} \rho^{n-2 s}$

where $n$ is the radial degree of the $j^{\text {th }}$ polynomial and $m$ its azimuthal frequency.

Let $\hat{\phi}(R \rho)$ be the estimated wavefront reconstructed by the adaptive optics system on the first $J$ polynomials from the measurements. In this paper, noise in the wavefront measurement will not be considered, therefore we write:

$\hat{\phi}(R \boldsymbol{\rho})=\sum_{j=2}^{J} a_{j} Z_{j}(\boldsymbol{\rho})$.

The residual phase variance $\epsilon^{2}(J, \alpha)$ is estimated for an adaptive optics system having $J$ degrees of freedom and for an angular distance $\alpha$ between the two directions, calculating:

$\epsilon^{2}(J, \alpha)=\int \mathrm{d}^{2} \boldsymbol{\rho} W(\boldsymbol{\rho})<(\phi(R \boldsymbol{\rho}, \alpha)-\hat{\phi}(R \boldsymbol{\rho}))^{2}>$. 
Using the orthogonality properties of Zernike polynomials, Eq. (1) and Eq. (6), and the stationarity hypothesis, the residual phase variance is:

$$
\begin{aligned}
\epsilon^{2}(J, \alpha) & =\sum_{j=J+1}^{\infty}<\left(a_{j}\right)^{2}>+2 \sum_{j=2}^{J}\left[<\left(a_{j}\right)^{2}>\right. \\
& \left.-<a_{j}(\alpha) a_{j}(0)>\right] .
\end{aligned}
$$

This result is classical in the estimation of wavefront residual error on the Zernike polynomial (Chassat 1989; Rousset 1993). The first term in brackets of the right hand side of Eq. (8) represents the fitting error for an adaptive optics system which corrects the first $J$ polynomials. It has been given by Noll (Noll 1976). The other term of the right hand side of the Eq. (8) is the spatial and angular wavefront errors.

The residual error can be written as:

$$
\begin{aligned}
\epsilon^{2}(J, \alpha) & =\sum_{j=2}^{\infty}\left\langle\left(a_{j}\right)^{2}\right\rangle \\
& -\sum_{j=2}^{J} \underbrace{\left[2<a_{j}(\alpha) a_{j}(0)>-<\left(a_{j}\right)^{2}>\right]}_{A(j, \alpha)} .
\end{aligned}
$$

The quantity $A(j, \alpha)$ is the spatial and angular error for the corrected polynomial $Z_{j}$. Correction by the adaptive optics system will be effective when $A(j, \alpha)$ is larger than 0 for each polynomial $Z_{j}$, i.e. when:

$$
\frac{<a_{j}(\alpha) a_{j}(0)>}{<\left(a_{j}\right)^{2}>} \geq 0.5 \text {. }
$$

When the criterion of Eq. (10) is satisfied, the measured wavefront at angular distance $\alpha$ provides a satisfactory fit of the on-axis wavefront when the correlation criterion of Eq. (10) is verified for all the correction modes. For instance, this criterion allows to select the number of useful correction modes for a given angular distance between the two wavefronts. Keeping in mind that all the low orders must be first corrected, a higher order mode will be corrected only if the criterion is verified. This result is general in wavefront residual error estimation for adaptive optics using the modal analysis (Rousset 1993).

Now, the Zernike coefficient angular correlations $<a_{j}(\alpha) a_{j}(0)>$ between the two wavefront directions can be derived as indicated in References (Valley \& Wandzura 1979; Chassat 1989; Molodij \& Rousset 1997). Therefore, the Zernike coefficient angular correlation can be written as:

$$
\begin{aligned}
<a_{j}(\alpha) a_{j}(0)>= & 3.895(n+1)\left(\frac{D}{r_{0}}\right)^{\frac{5}{3}} \\
& . \frac{\int_{0}^{L_{\mathrm{atm}}} \mathrm{d} h C_{n}^{2}(h) I_{n, m}\left(\frac{\alpha h}{R}\right)}{\int_{0}^{L_{\mathrm{atm}}} \mathrm{d} h C_{n}^{2}(h)}
\end{aligned}
$$

where $r_{0}$ in this equation is the Fried parameter, $D$ is the telescope diameter $(D=2 R), C_{n}^{2}$ is the turbulence profile, $\mathrm{h}$ is the altitude along the propagation path from $L_{\text {atm }}$ (the effective altitude of the considered atmosphere is 20000 meter) to the telescope, and using the notation $x=\frac{\alpha h}{R}$ :

$$
\begin{aligned}
I_{n, m}(x) & =s_{n, m} \int_{0}^{\infty} \mathrm{d} K K^{-\frac{14}{3}} J_{n+1}^{2}(K)\left[J_{0}(K x)\right. \\
& \left.+k_{j} J_{2 m}(K x)\right]
\end{aligned}
$$

where $K$ is the Fourier space of the Zernike polynomials in polar coordinates (Noll 1976). and

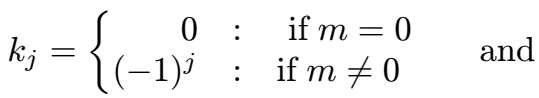

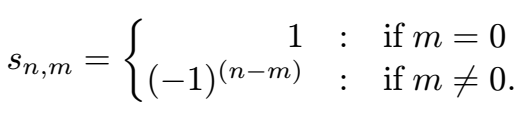

Let us notice that it is also possible to deduce from Eq. (11) and (12), the Zernike polynomial coefficient variances. The result is in perfect agreement with the variances given by Noll (Noll 1976). Let us remark that the variance only depends on the radial degree $n$ and is given by:

$<\left(a_{j}\right)^{2}>=3.895(n+1)\left(\frac{D}{r_{0}}\right)^{\frac{5}{3}} I_{n, 0}(0)$

where $I_{n, 0}(0)=\int_{O}^{\infty} \mathrm{d} K K^{\frac{-14}{3}} J_{n+1}^{2}(K)$.

To calculate the residual phase variance Eq. (8), we consider first that the number $J$ of corrected polynomials is chosen in order to include all the polynomials of the maximum radial degree $N$. Knowing that there is $n+1$ polynomials per radial degree $n$, we have therefore: $J=\frac{(N+1)(N+2)}{2}$.

Secondly, the azimuthal frequency symmetry properties of Zernike coefficient correlations are useful. Summation on all azimuthal frequencies of a radial degree leads to only a radial degree dependence. Then, we have using Eq. (11):

$$
\begin{aligned}
& \epsilon^{2}(N, \alpha)=3.895\left(\frac{D}{r_{0}}\right)^{\frac{5}{3}}\left[\sum_{n=1}^{\infty}(n+1)^{2} I_{n, 0}(0)-\sum_{n=1}^{N}(n+1)^{2}\right. \\
& \left.\left\{2 \frac{\int_{0}^{L_{\text {atm }}} \mathrm{d} h C_{n}^{2}(h) I_{n, m}\left(\frac{\alpha h}{R}\right)}{\int_{0}^{L_{\mathrm{atm}}} \mathrm{d} h C_{n}^{2}(h)}-I_{n, 0}(0)\right\}\right]
\end{aligned}
$$

Integral $I_{n, m}(x)$ (Eq. (12)) can be evaluated in closed form using Mellin transform techniques as proposed by Sasiela and expressed in terms of rapidly converging series (Sasiela 1994). This integral is transformed into a MellinBarnes integral and is then expressed as a sum of generalised hypergeometric functions in the appendix A. As mentioned by Tyler (Tyler 1990), this analytic form is 
related to integral involving product of three Bessel functions and can be evaluated only for restrictive cases, i.e. here depending on the angular separation. To overcome this limitation, Chassat (Chassat 1992) proposed an analytic solution applying a recursive development which is also presented in the Appendix.

\section{Problem of wavefront slope measurement}

In the previous paper (Molodij et al. 1996), we have presented a new tracking method, called granulation tracking, for measuring image motions by which extensive sources can control a tiltable mirror in real-time. The principle of this method is to monitor the variation of the image Fourier transform with respect to some reference, in order to estimate its motions. The image is detected by two detectors (Reticon $128 \mathrm{~s}$ photo-diode arrays), which cover a square field together but which each have resolution in one direction only. The reticon has elongated $2500 \times 25$ micron pixels that are sensitive to motion in the direction of the smaller diode dimension. Therefore, in the granulation image, the cross-section of the two arrays defines a square field of view which can be adjusted from $2 \times 2$ arcsecond to $12 \times 12$ arcsecond, depending on the Fried parameter $r_{0}$ (Rayrole 1992).

This tracking method, as in correlation tracking method, uses extended solar granulation images as target structure to detect wavefront aberrations. The size of the analyzed field of view is adjusted so the tracker will successfully operate under any seeing conditions. One has to keep in mind, however, that wavefront slope measurements are made to correct pure wavefront tilts. All higher order aberrations such as coma Zernike polynomials $\left(Z_{7}\right.$ or $\left.Z_{8}\right)$ are interpreted by the system as image motions because it is not possible to distinguish between, for example, a tilt in the wavefront and deformations of the object itself. The granulation tracker can not determine the appropriate tilts of the wavefront surface (which is the best correction in term of Strehl resolution). Such wavefront analyzer system determine relative displacements of successive images in real time. These shifts constitute arbitrary position of stabilization defined both by the target motions and the target structure deformations, which are applied to the tilting mirror.

We propose to analyze the problem of the wavefront slope measurement calculating the first phase derivative expansion on the Zernike polynomials using Primot's approach (Primot et al. 1990) in order to determine the image quality after image stabilization from wavefront slope measurements.

The wavefront slope measurements (in $x$ and $y$ direction) can be written:

$$
\left\{\begin{array}{l}
\frac{\delta \phi(x, y)}{\delta x}=\sum_{j=1}^{\infty} a_{j} \frac{\delta Z_{j}}{\delta x}=\sum_{j=1}^{\infty} a_{j} \sum_{j^{\prime}=1}^{\infty} \gamma_{j j^{\prime}}^{x} Z_{j^{\prime}} \\
\frac{\delta \phi(x, y)}{\delta y}=\sum_{j=1}^{\infty} a_{j} \frac{\delta Z_{j}}{\delta y}=\sum_{j=1}^{\infty} a_{j} \sum_{j^{\prime}=1}^{\infty} \gamma_{j j^{\prime}}^{y} Z_{j^{\prime}}
\end{array}\right.
$$

where $\gamma_{j j^{\prime}}^{x}$ and $\gamma_{j j^{\prime}}^{y}$ are the matrices expressed by Noll (Noll 1976) for representing derivatives of Zernike polynomials as a linear combination of Zernike polynomials.

We can express the wavefront slope measurements over the circular aperture of the telescope. For instance, the wavefront slope in $x$ direction, denoted $\overline{\frac{\delta \phi}{\delta x}}$ is written:

$$
\overline{\frac{\delta \phi}{\delta x}}=\sum_{j=1}^{\infty} a_{j} \sum_{j^{\prime}=1}^{\infty} \gamma_{j j^{\prime}}^{x} \int_{0}^{2 \pi} \frac{\mathrm{d} \theta}{\pi} \int_{0}^{1} r^{\prime} \mathrm{d} r^{\prime} Z_{j^{\prime}}\left(r^{\prime}, \theta\right) .
$$

Let us notice that averaging the Zernike polynomials over the aperture of the telescope is zero if $j^{\prime}>1$ :

$$
\left\{\begin{aligned}
\int \mathrm{d} \boldsymbol{r} W(\boldsymbol{r}) Z_{j^{\prime}}\left(\boldsymbol{r}^{\prime}\right) & =0 \\
\int \mathrm{d} \boldsymbol{r} W(\boldsymbol{r}) Z_{1}\left(\boldsymbol{r}^{\prime}\right) & =1
\end{aligned}\right.
$$

So that:

$\frac{\overline{\delta \phi}}{\delta x}=\sum_{j=1}^{\infty} a_{j} \gamma_{j 1}^{x}=\sum_{l=0}^{\infty} a_{2 l+1,1} \sqrt{4(l+1)}$

where the notation $Z_{j}(n=2 l+1, m=1)$ is adopted to define the concerned Zernike polynomials (the radial degree is odd and the azimutal frequency $m=1$ because the averaging over the circular aperture). The measured quantity is quite different from pure tilt Zernike polynomials. Wavefront tilt $Z_{2}$ not only appear to define the first mean phase derivative in $x$ direction, but also the $3^{\text {rd }}$ order coma $Z_{8}$ and higher order aberrations such $Z_{16}, Z_{30}$, $Z_{46}, Z_{68}$ etc.. Wavefront tilt $Z_{3}$ and others Zernike polynomial aberrations as $Z_{7}, Z_{17}, Z_{29}, Z_{47}, Z_{69}$ etc. appear to define the first mean phase derivative in y direction.

Therefore, the estimated wavefront reconstructed by the system from the measurements can be written:

$$
\hat{\phi}(R \rho)=a_{\frac{\delta \phi}{\delta x}} Z_{2}+a \frac{\delta \phi}{\frac{\delta \phi}{\delta y}} Z_{3}
$$

with:

$$
\left\{\begin{array}{l}
a_{\frac{\delta \phi}{\delta x}}=\frac{1}{2} \frac{\overline{\delta \phi}}{\delta x} Z_{2}=\left[\frac{1}{2} \sum_{l=0}^{\infty} \sqrt{4(l+1)} a_{2 l+1,1}\right] Z_{2} \\
a_{\frac{\delta \phi}{\delta y}}=\frac{1}{2} \frac{\delta \phi}{\delta y} Z_{3}=\left[\frac{1}{2} \sum_{l=0}^{\infty} \sqrt{4(l+1)} a_{2 l+1,1}\right] Z_{3}
\end{array}\right.
$$

Let us remark that effects of anisoplanatism must be taken into account to understand all the effects that might arise when adjusting the analyzed field of view of the target from $2 \times 2$ arcsecond to $12 \times 12$ arcsecond. The statistical analysis of angular cross-correlation allows to determine the aberration strenghts of all the higher order aberrations participating in the modal expansion of the first phase derivative of Eq. (21) in such situation.

Figure 1 shows the normalised angular correlation functions of the first polynomials of the expansion concerned for the $0.9 \mathrm{~m}$ telescope diameter (T.H.E.M.I.S.). These correlations are calculated using the simulated $C_{n}^{2}$ turbulence profile (Fig. 2) from experimental measurements at Izaña site by Arcetri University (Barletti et al. 1973). For 


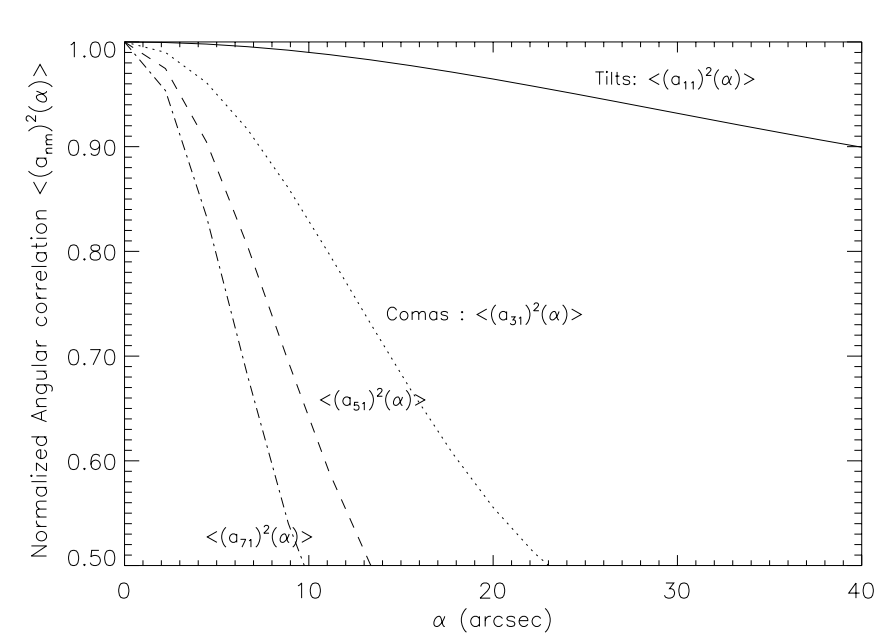

Fig. 1. Angular correlation functions for the higher order aberrations participating in the modal expansion of the first phase derivative versus the angular separation. The notation $Z_{j}(n=2 l+1, m=1)$ is adopted to define the concerned Zernike polynomials $j$ ( $n$ is the radial degree and $m$ the azimuthal frequency). These correlations are calculated using the simulated $C_{n}^{2}$ turbulence profile $\left(D / r_{0}=5\right)$ from experimental measurements at Izaña site by Arcetri University (Barletti et al. 1973)

a given angular separation $\alpha$ corresponding to the size of the field of view analyzed by the granulation tracker, the only Zernike polynomials defined by Eq. (21) which are useful in the measured quantity, are the polynomials having their $50 \%$ correlation angle larger than $\alpha$. The peculiar shape of the curves is related to the turbulence profile simulated, $\left(r_{0}=18 \mathrm{~cm}\right.$ of Fig. 2) (Barletti et al. 1973). The higher order aberrations participating in the modal expansion of the first phase derivative of Eq. (21) are correlated on the field of view delimitated by the granulation tracker so that the measured quantity by the analyzer is very close to the wavefront slope.

Let us notice that Fig. 1 reveals the strong dependence of the angular correlations on the angular separation $\alpha$ and the radial degree $n$. If the decorrelation of the tilts $(n=1)$ is slow when increasing $\alpha$, for others polynomials the decorrelation is steep. This result is general in the modal analysis of the effects of the atmospheric turbulence on the wavefronts (Rousset 1993).

Let us now consider how the correction is made by the image stabilizer system considering the residual wavefront error Eq. (7) when correcting pure wavefront tilts from slope measurements.

The estimated wavefront tilts by the system are given by Eq. (21) so that the residual error becomes:

$$
\epsilon^{2}(\alpha)=\sum_{n=1}^{\infty}(n+1)<\left(a_{n, 0}\right)^{2}>+2 \sum_{l=0}^{\infty} \sum_{l^{\prime}=0}^{\infty} \sqrt{(l+1)\left(l^{\prime}+1\right)}
$$

$$
<a_{2 l+1,1}(0) a_{2 l^{\prime}+1,1}(0)>-4 \sum_{l=0}^{\infty} \sqrt{l+1}<a_{2 l+1,1}(\alpha) a_{1,1}(0)>.
$$

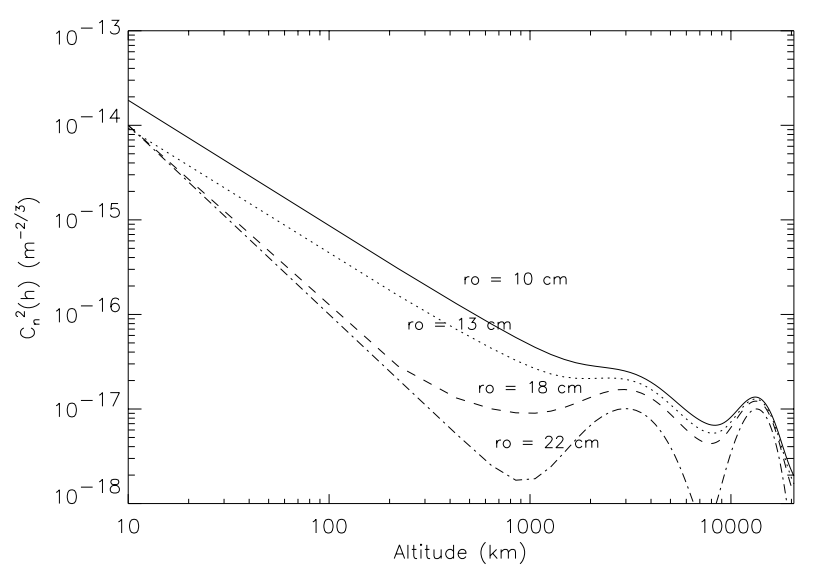

Fig. 2. $C_{n}^{2}$ turbulence profile from experimental measurements at Izaña site by Arcetri University (Barletti et al. 1973). The profiles are integrated from $10 \mathrm{~m}$ (above the ground) to $2000 \mathrm{~m}$ to give Fried's parameter $r_{0}$ at $\lambda=0.5 \mu \mathrm{m}$ from $10 \mathrm{~cm}$ to $22 \mathrm{~cm}$ (respectively $D / r_{0}$ from 9 to 4 )

The residual error after image stabilisation expansion contains angular cross-correlation of Zernike polynomials $j_{1}\left(n_{1}, m_{1}\right)$ and $j_{2}\left(n_{2}, m_{2}\right):<a_{n_{1}, m_{1}} a_{n_{2}, m_{2}}>$ which can be calculated introducing minor corrections in Eq. (11):

$$
\begin{aligned}
& <a_{j_{1}}(\alpha) a_{j_{2}}(0)>=3.895 \sqrt{\left(n_{1}+1\right)\left(n_{2}+1\right)}\left(\frac{D}{r_{0}}\right)^{\frac{5}{3}} \\
& \frac{\int_{0}^{L_{\text {atm }}} \mathrm{d} h C_{n}^{2}(h) I_{n_{1}+n_{2}, m_{1}+m_{2}}^{\prime}\left(\frac{\alpha h}{R}\right)}{\int_{0}^{L_{\text {atm }}} \mathrm{d} h C_{n}^{2}(h)}
\end{aligned}
$$

where $I_{n_{1}+n_{2}, m_{1}+m_{2}}^{\prime}(x)$ is now written:

$$
\begin{aligned}
& I_{n_{1}+n_{2}, m_{1}+m_{2}}^{\prime}(x)=s_{n_{1}+n_{2}, m_{1}+m_{2}} \sqrt{\left(n_{1}+1\right)\left(n_{2}+1\right)}\left[K_{1,2}^{+}\right. \\
& \int_{0}^{\infty} \mathrm{d} K K^{-14 / 3} J_{n_{1}+1}(K) J_{n_{2}+1}(K) J_{m_{1}+m_{2}}(K x)+K_{1,2}^{-} \\
& \left.\int_{0}^{\infty} \mathrm{d} K K^{-14 / 3} J_{n_{1}+1}(K) J_{n_{2}+1}(K) J_{\left|m_{1}-m_{2}\right|}(K x)\right]
\end{aligned}
$$

where $K_{1,2}^{+}$and $K_{1,2}^{-}$, quantities depending on the coefficient pairs $\left(j_{1}, j_{2}\right)$ parity, are listed in Table 1 .

The residual wavefront error after image stabilization may be expressed:

$\epsilon^{2}(1, \alpha)=3.895\left(\frac{D}{r_{0}}\right)^{\frac{5}{3}}\left[\sum_{n=1}^{\infty}(n+1)^{2} I_{n, 0}(0)\right.$ 
Table 1. Angular cross-correlation Zernike polynomial coefficients

\begin{tabular}{|l|l|l|l|}
\hline$K_{1,2}^{+}$ & $m_{1}=0$ & $m_{1} \neq 0 \& j_{1}$ even & $m_{1} \neq 0 \& j_{1}$ odd \\
\hline$m_{2}=0$ & 1 & $\sqrt{2}$ & 0 \\
\hline$m_{2} \neq 0 \& j_{2}$ even & $\sqrt{2}$ & 1 & 0 \\
\hline$m_{2} \neq 0 \& j_{2}$ odd & 0 & 0 & -1 \\
\hline$K_{1,2}^{-}$ & $m_{1}=0$ & $m_{1} \neq 0 \& j_{1}$ even & $m_{1} \neq 0 \& j_{1}$ odd \\
\hline$m_{2}=0$ & 0 & 0 & 0 \\
\hline$m_{2} \neq 0 \& j_{2}$ even & 0 & 1 & 0 \\
\hline$m_{2} \neq 0 \& j_{2}$ odd & 0 & 0 & 1 \\
\hline
\end{tabular}

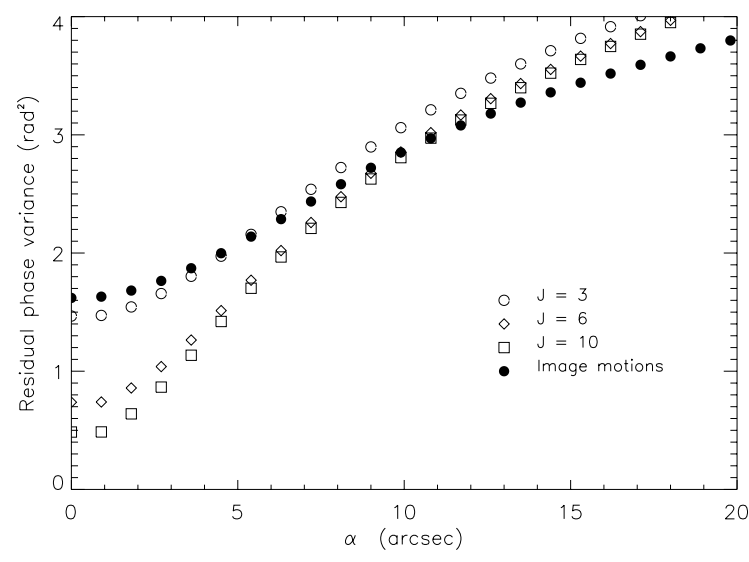

Fig. 3. Residual phase variance after compensation versus the angular separation $\alpha$. For comparison: residual phase variances after correction of $J=3$ polynomials $(N=1), J=6(N=2)$, $J=10(N=3)$ and after correction of pure tilts from wavefront slope measurements (dark points). Curves are calculated with the modelised profile at Izaña site $\left(D / r_{0}=4\right)$

$$
\begin{aligned}
& +2 \sum_{l=0}^{\infty} \sum_{l^{\prime}=0}^{\infty} \sqrt{(l+1)\left(l^{\prime}+1\right)(2 l+2)\left(2 l^{\prime}+2\right)} \\
& \times \frac{\int_{0}^{L_{\mathrm{atm}}} \mathrm{d} h C_{n}^{2}(h) I_{2 l+2 l^{\prime}+4,2}\left(\frac{\alpha h}{R}\right)}{\int_{0}^{L_{\mathrm{atm}}} \mathrm{d} h C_{n}^{2}(h)} \\
& -4 \sum_{l=0}^{\infty} \sqrt{(l+1) 2(2 l+2)} \\
& \left.\times \frac{\int_{0}^{L_{\mathrm{atm}}} \mathrm{d} h C_{n}^{2}(h) I_{2 l+2,2}\left(\frac{\alpha h}{R}\right)}{\int_{0}^{L_{\mathrm{atm}}} \mathrm{d} h C_{n}^{2}(h)}\right] .
\end{aligned}
$$

In any case the residual phase variance $\epsilon^{2}$ can be estimated from the knowledge of the Zernike coefficient angular correlations.

\section{Computation results}

The goal of this study is to compare the performance of the image stabilizer optical system and adaptive optics system which will correct aberrations as tilt, focusing, astigmatism and coma considering not only the image resolution but also the degradation of the compensation by the sys- tem in the field of view. Note that noise in the wavefront measurement will not be considered and the bandwidth of the servo-loop will be considered infinite.

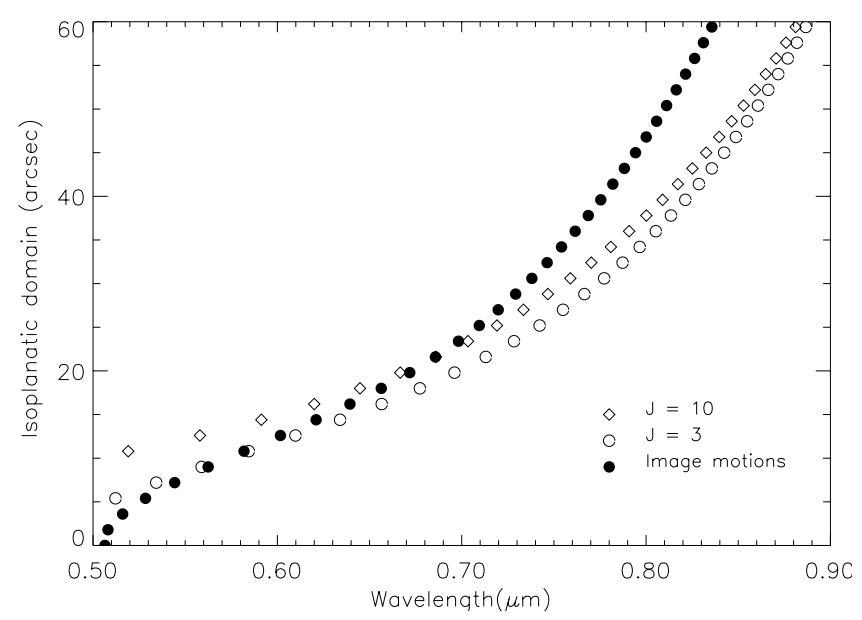

Fig. 4. Isoplanatic domain (twice as isoplanatic angle $\alpha_{\text {iso }}$ ) corresponding to an image quality criterion of $\frac{\lambda}{5}$, versus wavelength, for the T.H.E.M.I.S. telescope aperture $(D=0.9 \mathrm{~m})$ after compensation of $J=3$ first aberrated modes, $J=10$ modes and after image stabilisation (dark points). Curves are calculated with the modelised profile at Izaña site $\left(D / r_{0}=4\right)$

In order to determine the isoplanatic domain surrounding the target structure on-axis, we have to use a criterion to evaluate the quality of the restored image. This criterion is based on the residual phase variance, defined in Eq. (15) for the compensation of first aberrated modes and in Eq. (25) for image stabilizer system, which must be lower than a given value $\epsilon_{0}^{2}\left(\right.$ in $\left.\operatorname{rad}^{2}\right)$. An adaptive optics system compensating for turbulence disturbances up to the maximum radial degree $N(N=1$ for image stabilizer system) is characterized by the residual phase variance:

$\epsilon^{2}(N, \alpha)=\left(\frac{D}{r_{0}}\right)^{5 / 3} f(N, \alpha)$

where $f(N, \alpha)$ is deduced from Eq. (15) in the case of compensation of aberrated modes up to the maximum radial degree $N$ and from Eq. (25) in the case of image stabilizer system. The isoplanatic angle $\alpha_{\text {iso }}$ (half of the field of view) is given by the equality:

$\left(\frac{D}{r_{0}}\right)^{5 / 3} f\left(N, \alpha_{\text {iso }}\right)=\epsilon_{0}^{2}=\left(\frac{2 \pi}{a}\right)^{2} \quad\left(\operatorname{rad}^{2}\right)$.

Let us notice that the standard deviation $\Delta_{\mathrm{sd}}=\sqrt{\epsilon_{0}^{2}}$ may be expressed in wavelength unit: $\Delta_{\mathrm{sd}}=\frac{\lambda}{a}$ (rms).

In Fig. 3, the residual phase variances after compensation are plotted for T.H.E.M.I.S. telescope $(D=0.9 \mathrm{~m})$ and for the modelised profile at Izaña site defined by Fig. $2\left(r_{0}=22 \mathrm{~cm}\right)$. The curves correspond to different 
adaptive optics systems compensating for turbulence disturbances up to the maximum aberration modes $J$ (i.e. maximum radial degree $N$ ) compared to the image stabilizer system. These configurations are possible cases which could be considered for practical implementation (Kupke et al. 1994). Such type of curves have been first plotted by Chassat in order to manage optimal correction in terms of angular decorrelation by tuning up the number of corrected modes.

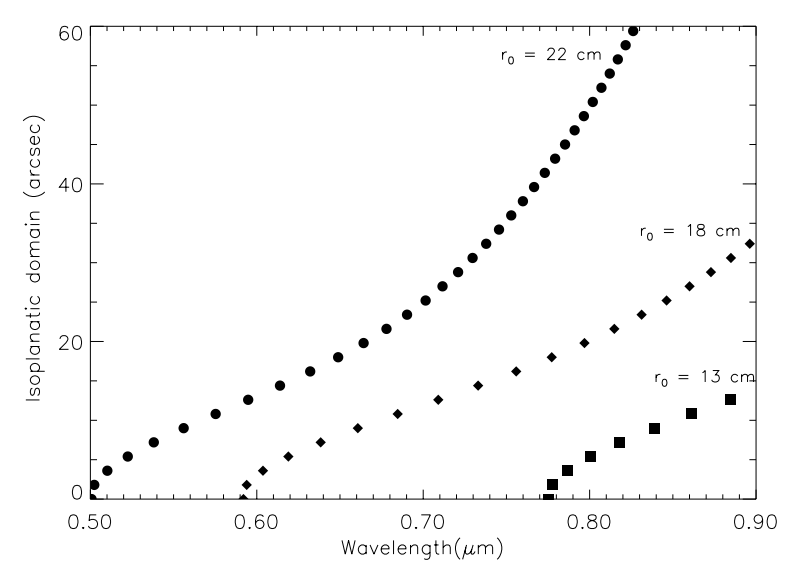

Fig. 5. Isoplanatic domain (twice as isoplanatic angle $\alpha_{\text {iso }}$ ) corresponding to an image quality criterion of $\frac{\lambda}{5}$, versus wavelength, for the T.H.E.M.I.S. telescope aperture $(D=0.9 \mathrm{~m})$ after image stabilisation. Curves are calculated with three modelised profiles from experimental measurements at Izaña site by Arcetri University (Barletti et al. 1973)

Notice that the $C_{n}^{2}$ profile, chosen to illustrate the derivations corresponds to a ratio $D / r_{0}=4$. In the three others modelised profiles of Fig. 2, the contribution of the turbulence near the ground increases or decreases both with the high altitude layers at $4000 \mathrm{~m}$ above the telescope altitude (and probably generated by the Pico del Teide). Therefore, the function $f(N, \alpha)$ in Eq. (26) remains identical when changing the ratio $D / r_{0}$. In such situations, the residual variance is given by a shift along the $y$ axis of Fig. 3 corresponding to the new value $\left(D / r_{0}\right)^{5 / 3}$.

First, let us underline the significant reduction of the residual phase variance on-axis $(\alpha=0)$ when increasing the number of corrected modes. The results shown by the curves $(J=3,6$ and 10) are in perfect agreement with the residual variance given by Noll (Noll 1976). The image motions compensation curve $(\alpha=0)$ reveals the contribution of the error made when correcting pure wavefront tilts from wavefront slope measurements.

Secondly, these results allow to understand that for small field of view observations, increasing the number of corrected mode is the best choice in terms of image quality (i.e. small phase variance) keeping in mind the fast degradation of this quality in the field. The obtained residual phase variance is very small after correction of 10 modes (point at $\alpha=0$ ). On the contrary, the on-axis correction after image stabilisation is relatively poor, but the residual phase variance does not vary rapidly with the field angle. At very large angle $(\alpha \geq 10$ arcsec), the correction after image stabilisation is even better than one obtained after correction of 10 aberrated modes, because of the low wavefront slope angular decorrelation. This demonstrates the importance of the decorrelation of the higher wavefront deformation modes in the field.

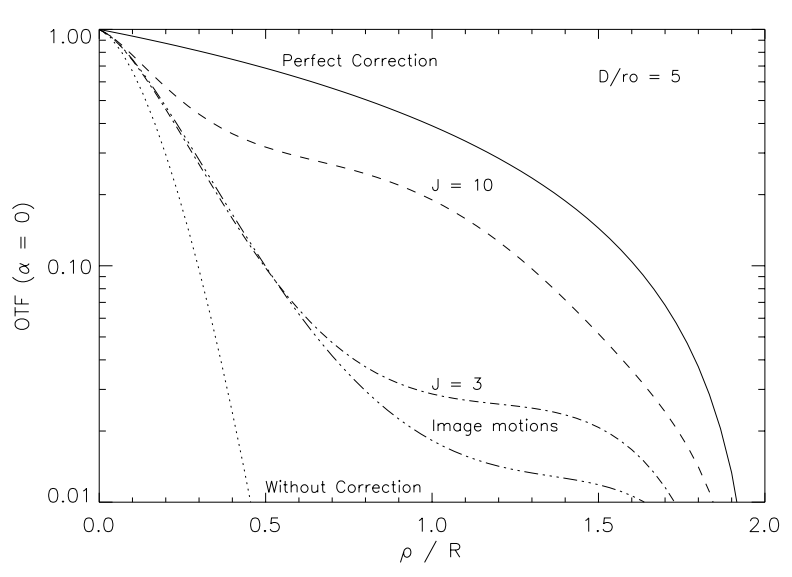

Fig. 6. $O T F(\alpha=0)$ versus the normalised spatial frequency $(\rho / R=2$ corresponds to the diffraction limited, i.e. a spatial resolution around $0.145 \operatorname{arcsec}$ at $\lambda=0.5 \mu \mathrm{m})$, for several orders of modal corrections (compensation through coma $J=10$, compensation of tilts $J=3$ and after image stabilization), at $D / r_{0}=5$. The full curve is the diffraction limited comparatively to the dot curve without correction

Using the relation between the wavelength and the residual phase variance, Eq. (27) can be written:

$\lambda(\mu \mathrm{m})=\frac{a}{2 \pi} \sqrt{f\left(\alpha_{\mathrm{iso}}\right)} 0.5\left(\frac{D}{r_{0}}\right)^{5 / 6}$

where $r_{0}$ is the Fried parameter calculated at the wavelength $\lambda=0.5 \mu \mathrm{m}$.

Figure 4 presents the isoplanatic angle $\alpha_{\text {iso }}$ for $\epsilon_{0}=2 \pi / 5$ (corresponding to $\lambda / 5$, i.e. a Strehl ratio around 20\%) calculated by Eq. (28). This value of residual error is somewhat arbitrary and rather a poor performance for an adaptive optics system but can be sufficient for many astronomical observations. As expected, the isoplanatic angle $\alpha_{\text {iso }}$ increases with the increase of the observation wavelength. Note that $\alpha_{\text {iso }}$ after image stabilisation is larger than $\alpha_{\text {iso }}$ after compensation of 10 first aberrated modes when observing at wavelength larger than $0.7 \mu \mathrm{m}$. Let us remind that for any field point at an angular distance to the target structure on-axis smaller or equal to $\alpha_{\text {iso }}$, the residual error is lower than $2 \pi / 5$. Depending on the choice 
of a maximum residual phase, the considered observation wavelength domain is not totally accessible (bandwidth from $0.5 \mu \mathrm{m}$ up to $0.9 \mu \mathrm{m}$ with T.H.E.M.I.S.). This is shown in Fig. 5 which present the isoplanatic domains after image stabilisation for three different values of Fried parameter $r_{0}$ obtained by the profile models (Fig. 2). For instance, with a Fried parameter $r_{0}=13 \mathrm{~cm}$, the observation wavelength must be larger than $\lambda_{\text {limit }}=0.78 \mu \mathrm{m}$ in order to achieve a residual error better than $\lambda / 5$. For $r_{0}=22 \mathrm{~cm}, \lambda_{\text {limit }}$ is $0.5 \mu \mathrm{m}$.

\section{Optical transfer functions}

The image quality obtained, after the T.H.E.M.I.S. adaptive optics system is applied, can be evaluated by comparing the restaured image frequencies of the optical tranfer function in the field of view. The optical transfer function after correction $(O T F)$ can be defined as the mathematical expectation of the cross-correlation function of the complex amplitude $\psi(\boldsymbol{r})$ of the compensated wavefront. Assuming that scintillating effects are negligible, this amplitude can be written:

$\psi(\boldsymbol{r})=\exp \left\{i \phi_{\mathrm{c}}(\boldsymbol{r})\right\}$

where the wavefront after compensation $\phi_{\mathrm{c}}(\boldsymbol{r})$ is the difference between the wavefront $\phi(\boldsymbol{r})$ coming from the observed source and the estimated wavefront $\hat{\phi}(\boldsymbol{r})$ reconstructed by the adaptive optics system from the measurement at angular distance $\alpha$.

Assuming the near field approximation is verified, the $O T F$ can be written:

$$
\begin{aligned}
O T F(\boldsymbol{\rho}, \alpha)= & \int \mathrm{d} \boldsymbol{r}<\psi\left(\boldsymbol{r}+\frac{\boldsymbol{\rho}}{2}\right) \psi^{*}\left(\boldsymbol{r}-\frac{\boldsymbol{\rho}}{2}\right)>W\left(\boldsymbol{r}+\frac{\boldsymbol{\rho}}{2}\right) \\
& . W\left(\boldsymbol{r}-\frac{\boldsymbol{\rho}}{2}\right)
\end{aligned}
$$

where $W(\boldsymbol{\rho})$ is the normalised function defined by Eq. (3). Having remarked that phase is a Gaussian random function (Roddier 1981), Eq. (30) becomes:

$$
\begin{gathered}
O T F(\boldsymbol{\rho}, \alpha)=\int \mathrm{d} \boldsymbol{r} \exp \left(-\frac{1}{2}<\left[\phi_{\mathrm{c}}\left(\boldsymbol{r}+\frac{\boldsymbol{\rho}}{2}\right)-\phi_{\mathrm{c}}\left(\boldsymbol{r}-\frac{\boldsymbol{\rho}}{2}\right)\right]^{2}>\right) \\
. W\left(\boldsymbol{r}+\frac{\boldsymbol{\rho}}{2}\right) W\left(\boldsymbol{r}-\frac{\boldsymbol{\rho}}{2}\right) .
\end{gathered}
$$

The statistics of the Zernike coefficients for an expansion of Kolmogorov turbulence phase distortion have been applied by Wang and Markey to calculate the $O T F$ for a point source case $(\alpha=0)$ (Wang \& Markey 1978). We have extended the method to cases when the observing source is extended in order to evaluate the degradation in the field of view of the image due to anisoplanatism after adaptive optics compensation.

Using Eqs. (1) and (6) for the wavefront expansions, the argument of the exponential of Eq. (31) can be expressed as:

$$
\begin{aligned}
E(\boldsymbol{r}, \boldsymbol{\rho})= & -\frac{1}{2}<\left[\phi\left(\boldsymbol{r}+\frac{\boldsymbol{\rho}}{2}\right)-\phi\left(\boldsymbol{r}-\frac{\boldsymbol{\rho}}{2}\right)\right]^{2}> \\
& -\frac{1}{2}<\left[\hat{\phi}\left(\boldsymbol{r}+\frac{\boldsymbol{\rho}}{2}\right)-\hat{\phi}\left(\boldsymbol{r}-\frac{\boldsymbol{\rho}}{2}\right)\right]^{2}> \\
& +<\left[\hat{\phi}\left(\boldsymbol{r}+\frac{\boldsymbol{\rho}}{2}\right)-\hat{\phi}\left(\boldsymbol{r}-\frac{\boldsymbol{\rho}}{2}\right)\right] \\
& {\left[\phi\left(\boldsymbol{r}+\frac{\boldsymbol{\rho}}{2}\right)-\phi\left(\boldsymbol{r}-\frac{\boldsymbol{\rho}}{2}\right)\right]>}
\end{aligned}
$$

We recognize in the right half of the equation the first term as the wave structure function. For a Kolmogorov type of turbulence, the wave structure function for a plane wave can be expressed:

$<\left[\phi\left(\boldsymbol{r}+\frac{\boldsymbol{\rho}}{2}\right)-\phi\left(\boldsymbol{r}-\frac{\boldsymbol{\rho}}{2}\right)\right]^{2}>=D(\boldsymbol{\rho})=6,88\left(\frac{\rho}{r_{0}}\right)^{5 / 3}$.

The cross-correlations of the Zernike coefficients $\left\langle a_{i} a_{j}\right\rangle$ $(\alpha)$ are calculated when the reference source target is at angular distance $\alpha$ from the observed source for $J$ corrected modes by the adaptive optics system (see Appendix). The turbulence profiles $C_{n}^{2}$ used in the calculation of the angular cross-correlation are presented in Fig. 2:

$<\left[\hat{\phi}\left(\boldsymbol{r}+\frac{\boldsymbol{\rho}}{2}\right)-\hat{\phi}\left(\boldsymbol{r}-\frac{\boldsymbol{\rho}}{2}\right)\right]^{2}>=\sum_{i=2}^{J} \sum_{j=2}^{J}<a_{i} a_{j}>H_{i, j}(\boldsymbol{r}, \boldsymbol{\rho})$

$<\left[\phi\left(\boldsymbol{r}+\frac{\boldsymbol{\rho}}{2}\right)-\phi\left(\boldsymbol{r}-\frac{\boldsymbol{\rho}}{2}\right)\right]\left[\hat{\phi}\left(\boldsymbol{r}+\frac{\boldsymbol{\rho}}{2}\right)-\hat{\phi}\left(\boldsymbol{r}-\frac{\boldsymbol{\rho}}{2}\right)\right]>=$

$\sum_{i=2}^{\infty} \sum_{j=2}^{J}<a_{i} a_{j}>(\alpha) H_{i, j}(\boldsymbol{r}, \boldsymbol{\rho})$.

Introducing the notation:

$$
\begin{aligned}
H_{i, j}(\boldsymbol{r}, \boldsymbol{\rho}) & =\left[Z_{i}\left(\boldsymbol{r}+\frac{\boldsymbol{\rho}}{2}\right)-Z_{i}\left(\boldsymbol{r}-\frac{\boldsymbol{\rho}}{2}\right)\right]\left[Z_{j}\left(\boldsymbol{r}+\frac{\boldsymbol{\rho}}{2}\right)\right. \\
& \left.-Z_{j}\left(\boldsymbol{r}-\frac{\boldsymbol{\rho}}{2}\right)\right] .
\end{aligned}
$$

The $O T F$ in the field of view is obtained evaluating the following numerical expression as indicated by Wang \& Markey (Wang \& Markey 1978):

$O T F(\boldsymbol{\rho}, \alpha)=\frac{4}{\pi} \int_{0}^{\pi / 2} \mathrm{~d} \theta \int_{0}^{r_{\max }} \mathrm{d} r r \exp [E(\boldsymbol{r}, \boldsymbol{\rho})]$

with

$\left.E(\boldsymbol{r}, \boldsymbol{\rho}) \simeq-\frac{1}{2} D(\boldsymbol{\rho})+\sum_{i=2}^{J} \sum_{j=2}^{J}\left[\left\langle a_{i} a_{j}\right\rangle(\alpha)-\frac{1}{2}<a_{i} a_{j}\right\rangle\right]$ 
$H_{i, j}(\boldsymbol{r}, \boldsymbol{\rho})+\sum_{i=J+1}^{J_{\text {limit }}} \sum_{j=2}^{J}<a_{i} a_{j}>(\alpha) H_{i, j}(\boldsymbol{r}, \boldsymbol{\rho})$

and

$r_{\max }=-\frac{\rho}{2} \cos (\theta-\varphi)+\sqrt{1-\left(\frac{\rho}{2} \sin (\theta-\varphi)\right)^{2}}$

where $\varphi$ is the argument of $\boldsymbol{\rho}$.

First, we have verified that the infinite sum can be stopped at $J_{\text {limit }}=91$ when the compensating phase distribution is an expansion through coma $J=10$ and $\alpha=0$, as indicated by Wang and Markey.

Secondly, when $\alpha \geq 10$ arcsec with the considered turbulence profile, the cross-correlation terms can be approximated by truncating the infinite sum over a more appropriate limit around $J_{\text {limit }}=40$ (for an estimated convergence at $10^{-8}$ ). This is due to the fact that the loss of cross-correlations of higher order aberrations is more important in the field of view (depending on $\alpha$ ) than the lower orders.

Figure 6 shows the $O T F(\alpha=0)$ versus the spatial frequency calculated with the turbulence profile presented in Fig. $2\left(r_{0}=18 \mathrm{~cm}\right)$ and zero field of view for 10 orders of correction (compensation through coma) and for 3 orders of correction (compensation of tilts) comparatively to image motion correction (image stabilization). The results obtained for $J=10$ and $J=3$ agree exactely with the analytical calculation reported by Wang and Markey. An analytic expression of the OTF taking into account the

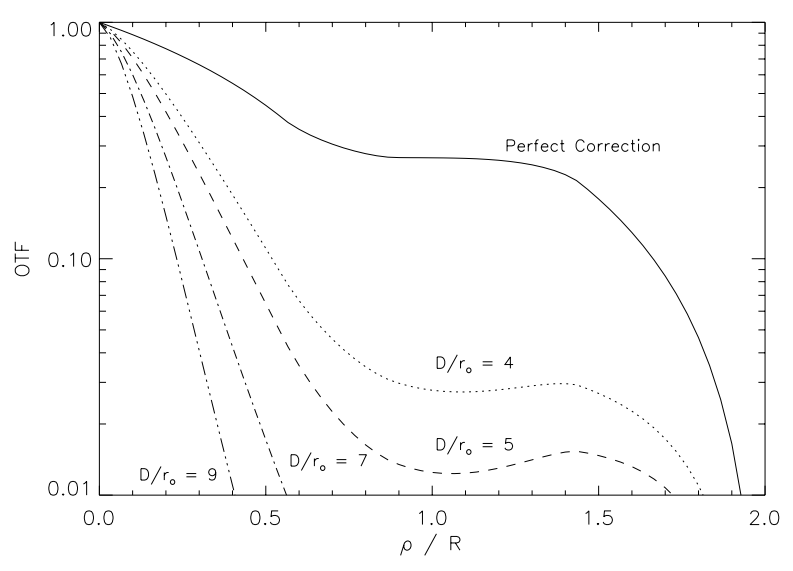

Fig. 7. $O T F(\alpha=0)$ versus the normalised spatial frequency $\rho / R$, for various level of turbulence taking into account the central obscuration of telescope T.H.E.M.I.S. $(u=d / D=0.44)$. The $C_{n}^{2}$ turbulence profiles used in the calculations, have been simulated from experimental measurements made at Izaña site. The full curve is the diffraction limited central obscuration of the telescope has been published by Perrier (Perrier 1989). Therefore, the OTF can be written:

$$
O T F_{\mathrm{co}}(\boldsymbol{\rho}, \alpha)=O T F(\boldsymbol{\rho}, \alpha) \cdot \frac{G(\boldsymbol{\rho}, u)}{O T F_{\mathrm{diffrac}}}
$$

where $u=\frac{d}{D}$ is the linear central obscuration by comparison to the T.H.E.M.I.S. diameter $D$. In this case $u=0.44$. Figure 7 presents the image quality improvement after compensation by the image stabilizer system taking into account the central obscuration of the magnetograph for various levels of turbulence and zero field of view. One observes that the image quality improvement grows when the turbulence strength expressed by Fried's parameter $r_{0}$ increases, i.e., when the ratio $D / r_{0}$ decreases. For instance, in Fig. 7 the image motion stabilization is insufficient for high turbulence levels caracterized by a ratio $D / r_{0} \geq 6$ when imaging in the visible wavelength $(\lambda=0.5 \mu \mathrm{m})$. High spatial frequencies are not restored by image stabilizer system compensation.

Effects of anisoplanatism are presented in Fig. 8. The $O T F$ for compensation through the coma $J=10$ are shown for different angular separations in the field of view for the same turbulence profile. As expected comparing with Fig. 4, one observes that the image quality improvement when compensating 10 modes decreases rapidly when the angular separation increases for an extended source. At very large angle $(\alpha \geq 20$ arcsec $)$ in Fig. 8, the image quality after compensation of 10 modes for $D / r_{0}=5$ is hardly better than the one obtained without correction, because of the wavefront angular decorrelation.

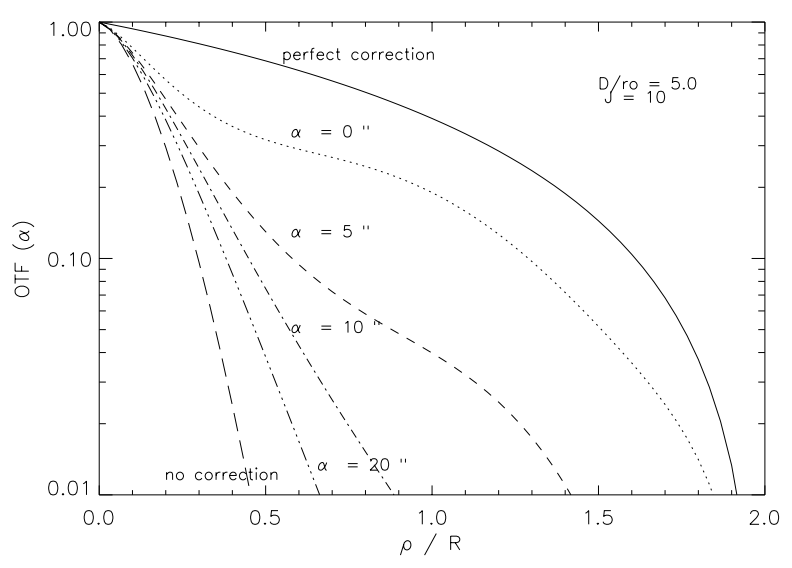

Fig. 8. $O T F(\alpha)$ versus the normalised spatial frequency $\rho / R$, for several angular separation $\alpha$ for a compensation by the adaptive optics system through the coma $J=10$. The full curve is the diffraction limited

In Fig. 9 the relative $O T F$ corresponding to the ratio of the $O T F$ after compensation through $J=10$ by the $O T F$ after compensation through $J=3$, OTF $=$ 


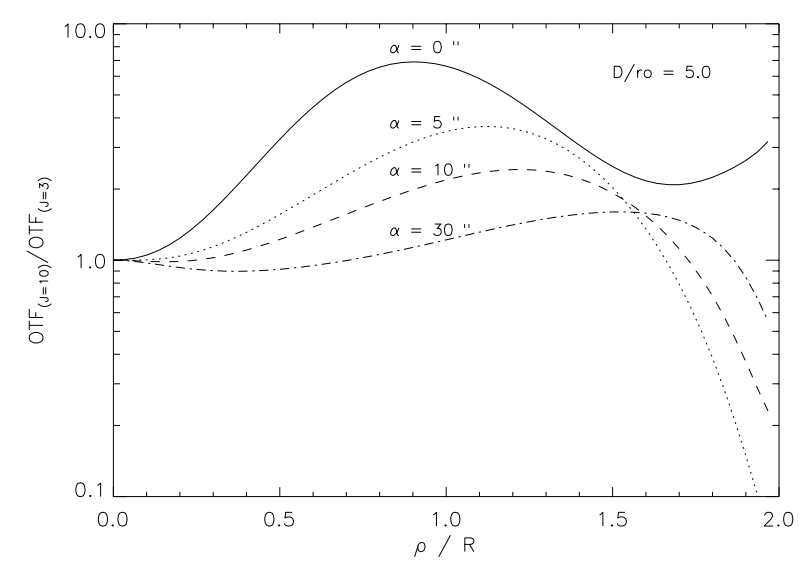

Fig. 9. Relative $O T F=O T F_{J=10} / O T F_{J=3}$ versus the normalised spatial frequency $(\rho / R)$ for several angular separations $\alpha$ in the field of view. Curves are calculated with the modelised profile $\left(r_{0}=18 \mathrm{~cm}\right.$ from experimental measurements at Izaña site by Arcetri University (Barletti et al. 1973)

$O T F_{J=10} / O T F_{J=3}$, are plotted versus the normalised spatial frequency for different angular separations $\alpha$. Figure 9 allows to understand the problem of the degradation of the compensation in the field of view due to anisoplanatism when increasing the order of correction from $J=3$ up to $J=10$. In the case of point source correction (curve $\alpha=0$ ), increasing the order of correction permits a higher-level image quality. In the case of an extended source ( $\alpha \geq 5$ arcsec for the turbulence profile studied), increasing the order of correction can be detrimental to the image quality. For instance, the curve $\alpha=5$ arcsec of Fig. 9 shows that higher spatial frequencies $(\rho / R \geq 1.4)$ are degraded when the compensating phase distribution is an expansion through coma comparatively to a tilts compensation by the adaptive optics system. This anisoplanatism effect can be understood by remarking that the loss of correlation is faster for the higher order coefficients than for low order. Of course, this behaviour is emphasized in the comparison between $O T F_{J=10}$ and $O T F_{J=3}$ because of the large isoplanatic patch for the tilt aberration. Similar conclusions were found by Abitbolt and Ben-Yosef (Abitbolt \& Ben-Yosef 1991).

We present the results of numerical simulations to analyze the effects of anisoplanatism when observing extended sources with possible configurations of adaptive optics system which could be considered for practical implementation on T.H.E.M.I.S. We compute analytically the long exposure optical tranfer functions $O T F$ in the field of view (for different angular separation $\alpha$ ) in order to simulate the filtering process of the atmospheric turbulence on the spatial frequencies of a long exposure image of the solar granulation.

Figure 10 demonstrates the necessity to use adaptive optics system to compensate the terrestrial atmospheric perturbations on wavefronts coming from the Sun to the telescope (comparison between Fig. 10a and Fig. 10b). Figure 10a shows a $15 \operatorname{arcsec}^{2}$ field of view of the solar granulation obtained with short exposures $(\simeq$ few $\mathrm{ms}$ in imagery mode). This image allows to simulate the effects of atmospheric turbulence in cases when long exposure are indispensable for accurate measurements in spectroscopy mode $(\simeq 300 \mathrm{~ms})$ before and after compensation. A comparison of the effects of anisoplanatism is shown on an extended image of solar granulation after real time compensation by the image stabilizer system and by a 10 controlable modes adaptive optics system (Fig. 10c and Fig. 10a).

Let us underline the significant improvement of the image quality when compensating the 10 first aberration modes on this small field of view of the image granulation (Fig. 10d). By comparison, the on-axis correction is relatively good with the image stabilizer optical system. It reveals the importance of the contribution of the tilts in the adaptive optics compensation when observing with a $90 \mathrm{~cm}$ telescope aperture for mean atmospheric turbulences. Notice also that tilt variances represent $90 \%$ of the total variance phase (Noll 1976).

Figure 11 presents the same comparison on a very large field of view (around $50 \operatorname{arcsec}^{2}$ ). One observes the significant increase of anisoplanatism effects in the field of view with the increase of the number of corrected modes (Fig. 11c: image stabilization with a tip-tilt mirror, Fig. 11d: compensation through the coma $J=10$ ). This simulation allows to understand that for small field of view observations, compensation of the 10 first aberration modes is the best choice in terms of image quality keeping in mind the fast degradation of this quality in the field of view as shown by Fig. 11d. In contrary, with image stabilization, the image quality does not vary fastly with the field angle. It reveals the important contribution of the large isoplanatic patch of the tilt aberrations for the adaptive optics compensation. At very large angle $(\alpha \geq$ $20 \operatorname{arcsec})$, the correction with the image stabilizer optical system (Fig. 11c) is even better than the one obtained with the compensation of 10 first aberration modes by adaptive optics system (Fig. 11d).

Figure 12a demonstrates the capacity of image stabilizer system for large field of view observations and medium image quality. The on-axis correction is relatively good considering the evaluation of the Fried's resolution (0.45 arcsec in the center part of the field of view) and the degradation does not vary fastly with the field angle. This is due to the dominant contribution of the lawest altitude layers in the angular correlation of the wavefronts. This system is well designed for solar observations like sunspots (up to 30 arcsec), active regions and protuberances (few arcminutes). Notice that the temporal analysis of solar phenomena needs also an homogeneous field of view. 


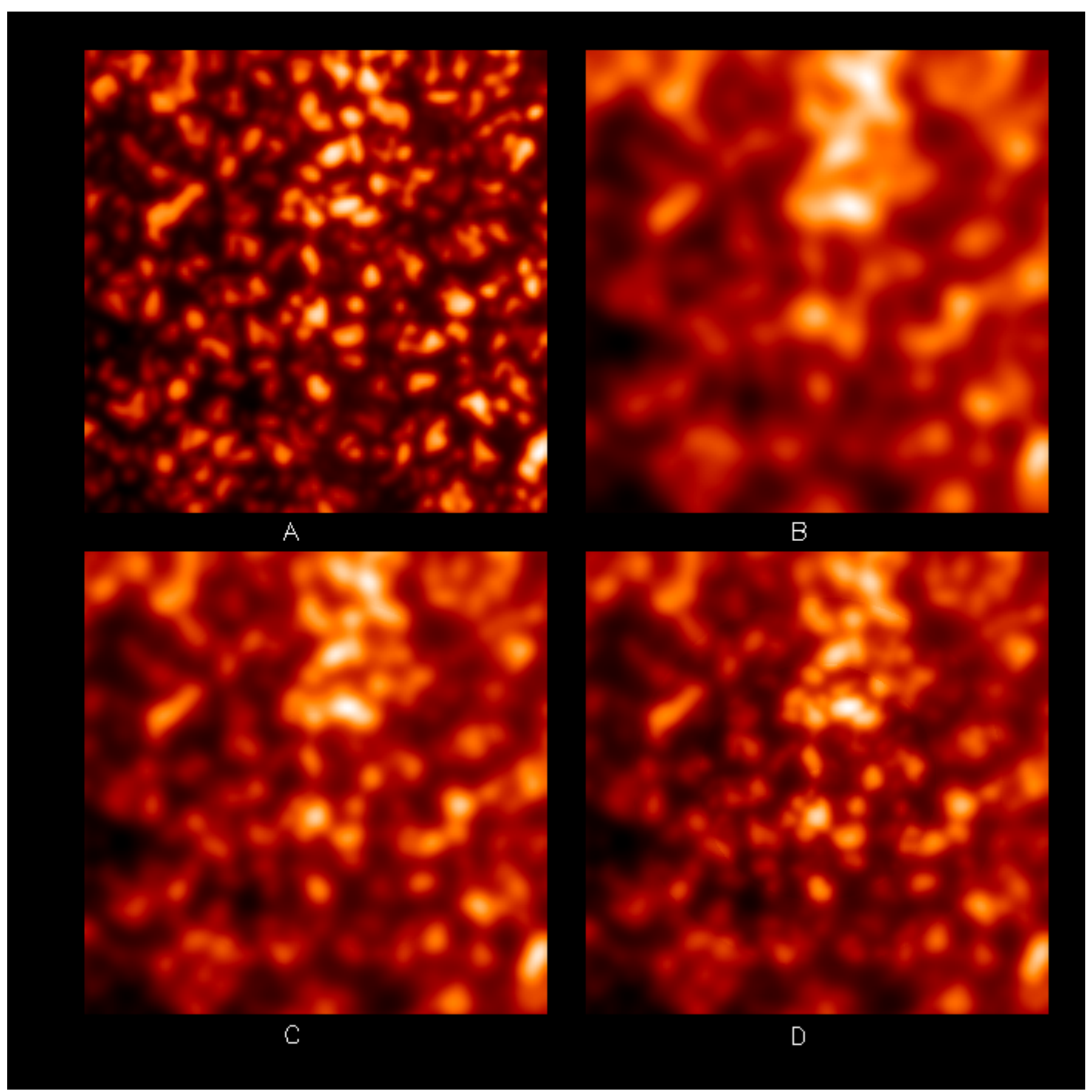

Fig. 10. A) Solar granulation at diffraction (short exposure). Image obtained by R. Muller. The size of the field-of-view is $15 \times 15$ arcsec. B) Effects of the turbulence for long exposure. The Fried's resolution is around $0.8 \operatorname{arcsec}\left(r_{0}=18 \mathrm{~cm}\right)$. The turbulence profile has been simulated from experimental measurements at Izaña site by Arcetri university (Barletti et al. 1973). C) Anisoplanatism effects after real-time compensation by the image stabilizer optical system. D) Anisoplanatism effects after correction of 10 controlable modes (tilt, focusing, astigmatism and coma) 


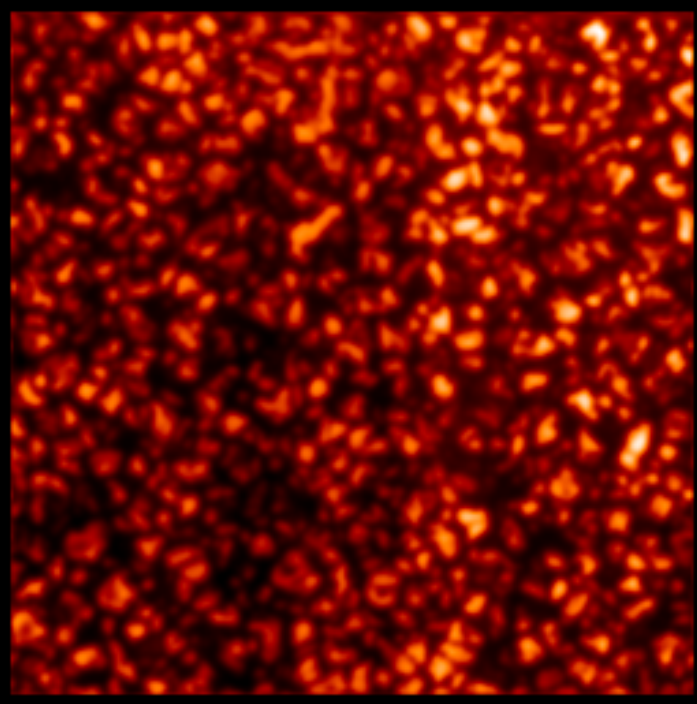

A

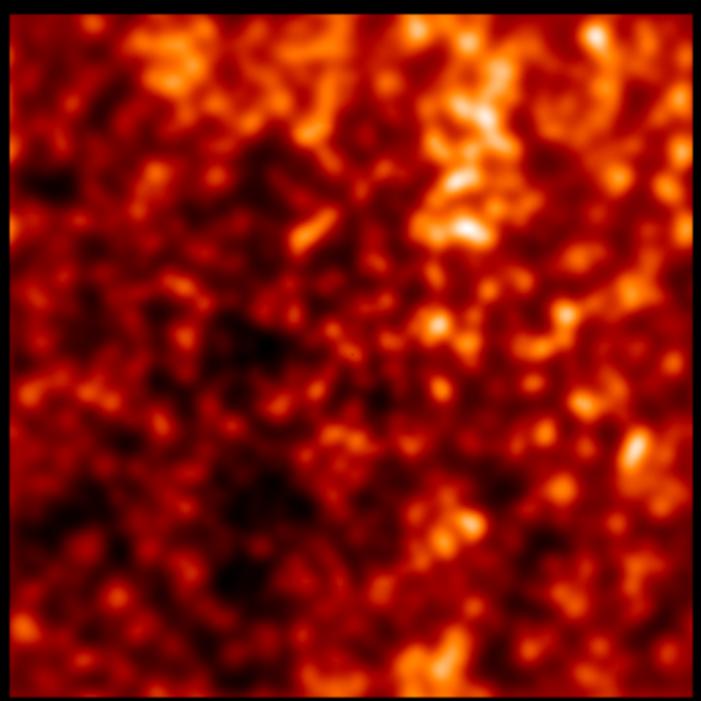

$\mathrm{C}$

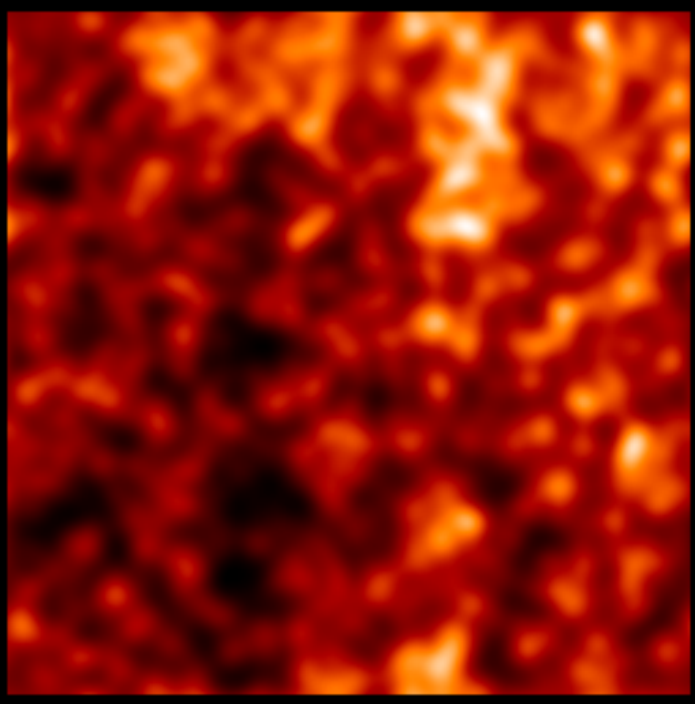

B

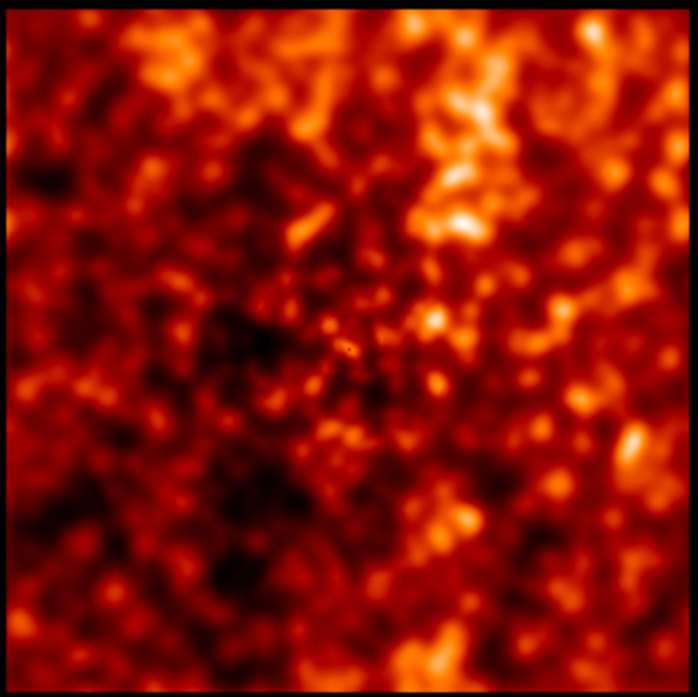

D

Fig. 11. A) Solar granulation at diffraction (short exposure). Image obtained by R. Muller. The size of the field-of-view is $50 x 50$ arcsec. B) Effects of the turbulence for long exposure. The Fried's resolution is around $0.8 \operatorname{arcsec}\left(r_{0}=18 \mathrm{~cm}\right)$. The turbulence profile has been simulated from experimental measurements at Izaña site by Arcetri university (Barletti et al. 1973). C) Anisoplanatism effects after real-time compensation by the image stabilizer optical system. D) Anisoplanatism effects after correction of 10 controlable modes (tilt, focusing, astigmatism and coma) 

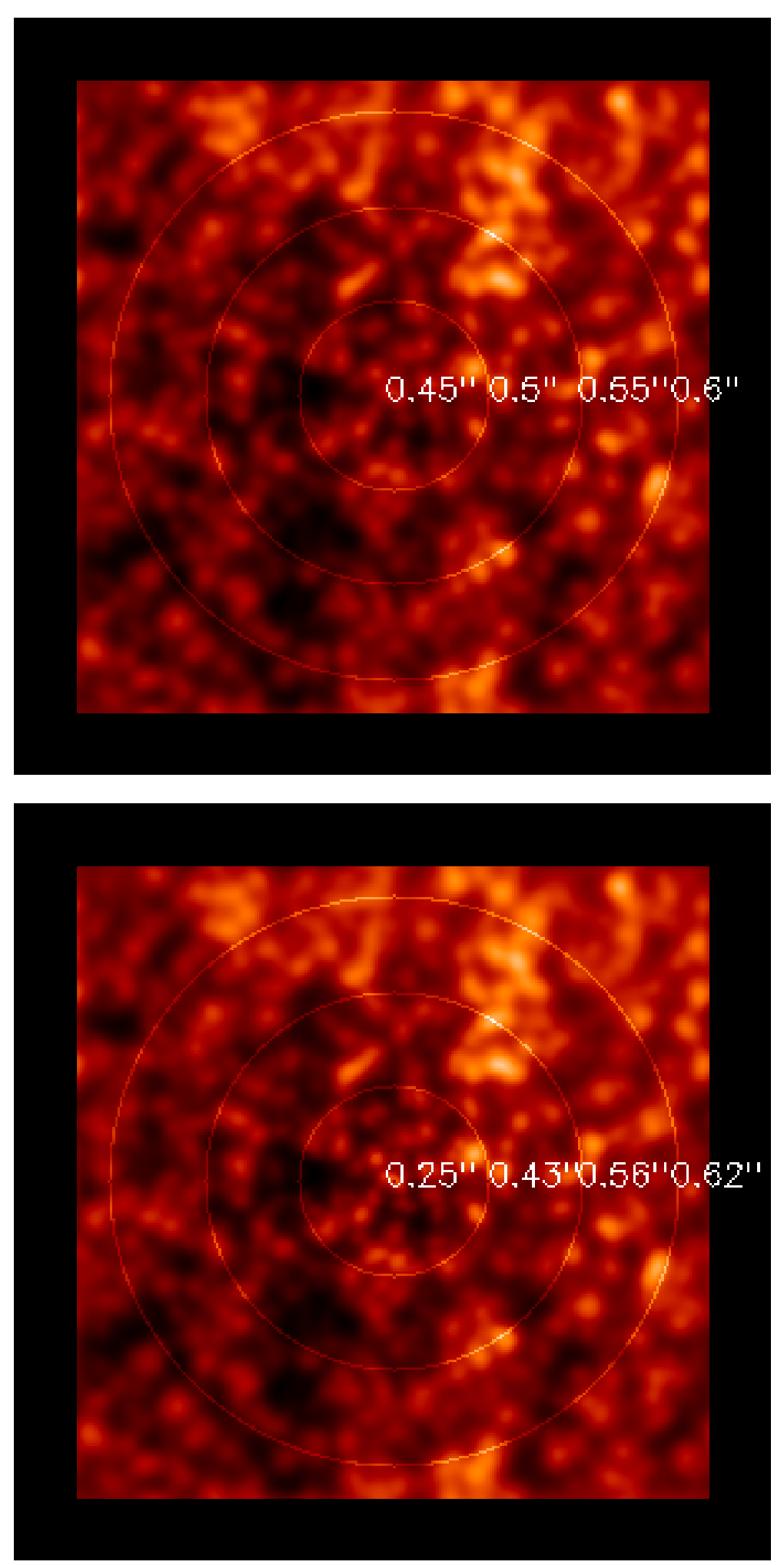

Fig. 12. Evaluation of Fried resolution in the field-of-view ( $50 \mathrm{arcsec})$ for image stabilizer system (top: A) and (bottom: B) for adaptive optics system after correction of 10 controlable modes (tilt, focusing, astigmatism and coma). By comparison, the resolution without correction is only $0.8 \operatorname{arcsec}\left(r_{0}=18 \mathrm{~cm}\right)$ 
By comparison, Fig. 12b allows to understand that for small field of view observations, the 10 corrected modes adaptive optics system is the best choice in terms of image quality keeping in mind the fast degradation of the quality in the field (resolution of 0.25 arcsec up to 0.62 arcsec in the 50 arcsec field). This demonstrates the importance of the decorrelation of the higher wavefront deformation modes in the field. So, the fast degradation of the resolution in the field will be problematic for many astronomical observations. Nevertheless, this system will be well designed to the observation of concentrated structures of the magnetic field $(0.2$ arcsec), penumbra fibrils (0.5 arcsec) or details of sunspots.

\section{Conclusion}

The generalized theoretical analysis of correlation functions leads to an accurate modelling of anisoplanatism. The residual wavefront error derivation presented in Sect. 2 and 4 provides a useful analysis to evaluate the effects of anisoplanatism upon the performance of solar adaptive optics systems. The theoretical analysis uses the wavefront expansion on the Zernike polynomials which is also used in Sect. 3 to analyze the problem of the wavefront slope measurements on the granulation image when compensating with a tiltable mirror. A detailed evaluation of the anisoplanatism is deduced from the Zernike polynomial angular correlations, derived using the Mellin transform technique. In Sect. 5, the optical transfer functions are evaluated for a Zernike expansion following the Wang and Markey approach. The calculation of OTF to cases when the observing source is extended is developed in order to evaluate the degradation in the field of view of the image due to anisoplanatism after adaptive optics compensation.

We compute the isoplanatic angle corresponding to a $\lambda / 5$ maximum wavefront error in the field of view for T.H.E.M.I.S. after compensation by the image stabilizer optical system and different observation wavelengths. Depending on the level of the strength of the turbulence, the accessible wavelength range is limited. We compare the performances of the actual image stabilizer optical system and a project of 10 controlable modes of correction (tilt, focusing, astigmatism and coma). We demonstrate the capacity of 10 controlable corrected modes for high quality compensation but to the price of a small field of view while the capacity of the image stabilizer optical system is for medium quality compensation and large field of view observations.

The study may be useful for the design of future adaptive optics system for solar observations. Depending of the specific goals, large or small field of view and medium or high image quality, the system may be totally different.

The authors are grateful to R. Muller for granulation images of the sun from the Pic-du-midi Observatory.

\section{A. Appendix}

As demonstrated by Sasiela (Sasiela 1994), the properties of Mellin transforms (see for instance (Colombo 1959; Dautray \& Lions 1987)) and Gamma functions are useful in problems dealing with wave propagation in turbulence. This technique is applied here to solve the integral in Eq. (12), Eq. (24), Eq. (34) and Eq. (35).

The Mellin transform pair is given by:

$$
\begin{aligned}
& F(s)=\int_{0}^{\infty} \mathrm{d} K K^{s-1} f(K) \quad \text { and } \\
& f(K)=\frac{1}{2 i \pi} \int_{\sigma-\infty}^{\sigma+\infty} \mathrm{d} s K^{-s} F(s) .
\end{aligned}
$$

The general form of the integral to solve has been presented by Chassat (Chassat 1989) and can be written $\left(\eta=\frac{14}{3}\right)$ :

$$
I(x)=\int_{0}^{\infty} \mathrm{d} K K^{-\eta} J_{\alpha}(K) J_{\gamma}(K) J_{\beta}(x K) .
$$

From the properties of the Mellin transforms (Colombo 1959; Dautray \& Lions 1987) and using $F_{n}(s)=$ $\int_{0}^{\infty} \mathrm{d} K K^{s-1} J_{n}(K)$, the integral becomes:

$$
\begin{aligned}
I(x) & =\frac{1}{(2 i \pi)^{2}} \int_{c-\infty}^{c+\infty} \int_{c-\infty}^{c+\infty} F_{\alpha}(s) F_{\beta}(t) x^{-t} F_{\gamma} \\
& \times(-\eta-t+1-s) \mathrm{d} s \mathrm{~d} t .
\end{aligned}
$$

Tables of Mellin transforms (Sasiela 1994; Colombo 1959; Dautray \& Lions 1987) are helpful to solve Eq. (A3). Moreover, the Mellin transform of functions can usually be expressed as the ratio of Gamma functions. We use the notation:

$\Gamma\left[\begin{array}{l}x_{1}, x_{2}, \ldots, x_{n} \\ y_{1}, y_{2}, \ldots, y_{m}\end{array}\right]=\frac{\Gamma\left(x_{1}\right) \Gamma\left(x_{2}\right) \ldots \Gamma\left(x_{n}\right)}{\Gamma\left(y_{1}\right) \Gamma\left(y_{2}\right) \ldots . \Gamma\left(y_{m}\right)}$.

We have for $J_{n}$ the $(n)^{\text {th }}$ order Bessel function of the first kind:

$F_{n}(s)=2^{s-1} \Gamma\left[\begin{array}{l}\frac{s}{2}+\frac{n}{2} \\ \frac{n}{2}-\frac{s}{2}+1\end{array}\right]$

with $\operatorname{Re}(-n)<\operatorname{Re}(s)<\frac{3}{2}$.

It leads to the Mellin-Barnes integral:

$I(x)=\frac{1}{(2 i \pi)^{2}} \int_{-i \infty}^{+i \infty} \int_{-i \infty}^{+i \infty} 2^{-\eta} x^{-2 t}$
$\Gamma\left[\begin{array}{l}-s-t+\frac{\alpha-\eta+1}{2}, t+\frac{\beta}{2}, s+\frac{\gamma}{2} \\ 1+t+s+\frac{\alpha+\eta-1}{2}, 1-t+\frac{\beta}{2}, 1-s+\frac{\gamma}{2}\end{array}\right] \mathrm{d} s \mathrm{~d} t$.

This integration can be performed using the method of pole residues. The value of the integral, as given by 
Cauchy's formula, is just $2 \mathrm{i} \pi$ times the sum of the residues at the enclosed poles. The result can be expressed in terms of generalised hypergeometric functions (Abramovitz \& Stegun 1965). For instance, in the case $x=\frac{\alpha h}{R}>1$, one of the interesting cases, the integral is given by:

$I(x)=F_{4}\left[\frac{1}{2}\left(\alpha+\gamma+\beta-\frac{11}{3}\right), \frac{1}{2}\left(\alpha+\gamma-\beta-\frac{11}{3}\right), \alpha+1\right.$,

$\left.\gamma+1 ; \frac{1}{x^{2}}, \frac{1}{x^{2}}\right] \frac{\Gamma\left[\frac{1}{2}\left(\alpha+\delta+\beta-\frac{11}{3}\right]\right.}{\Gamma[\gamma+1]} \frac{\Gamma\left[\frac{1}{2}\left(\alpha+\gamma-\beta-\frac{11}{3}\right]\right.}{\Gamma[\alpha+1]}$

$\frac{(-1)^{2 p+2 q}}{-\pi} 2^{-\frac{11}{3}} x^{-n_{i}-n_{j}+\frac{5}{3}} \sin \left(\frac{\pi}{2}\left(\alpha+\gamma-\beta-\frac{17}{3}\right)\right)($

where:

$F 4\left(\alpha, \beta, \gamma, \gamma^{\prime} ; x, y\right)=\sum_{m=0}^{\infty} \sum_{n=0}^{\infty} \frac{(\alpha)_{m+n}(\beta)_{m+n} x^{m} y^{n}}{(\gamma)_{m}\left(\gamma^{\prime}\right)_{n} m ! n !}$

with the notation $(a)_{n}=\frac{\Gamma(a+n)}{\Gamma(a)}$.

When the function is expressed in terms of generalised hypergeometric functions, the integral leads to the more restrictive convergence condition which can be expressed as: $\frac{\alpha h}{R}<2$. This condition determines the maximum field of view which can be reached by this method.

The limited convergence domain encountered in the evaluation of integrals involving the product of three Bessel functions has been first remarked by Tyler (Tyler 1990). Chassat proposed a method using the Bessel recurrency law (Chassat 1992):

$J_{\mu+2}(K)=\frac{2(\mu+1)}{K} J_{\mu+1}(K)-J_{\mu}(K)$.

Two easily evaluable Mellin integrals appear, $I_{1}(x, \mu, \beta, \eta)$ and $I_{2}(x, \mu, \beta, \eta)$

$I_{1}(x, \mu, \beta, \eta)=\int_{0}^{\infty} \mathrm{d} K K^{-\eta} J_{\mu}^{2}(K) J_{\beta}(K x)$

$I_{2}(x, \mu, \beta, \eta)=\int_{0}^{\infty} \mathrm{d} K K^{-\eta} J_{\mu}(K) J_{\mu+1}(K) J_{\beta}(K x) .($

With the notation $\mu=\inf (\alpha, \gamma)$ and $\epsilon=|\alpha-\gamma|$, Eq. (A2) becomes:

$$
\begin{aligned}
I(x) & =\int_{0}^{\infty} \mathrm{d} K K^{-\eta} J_{\mu} J_{\mu+\epsilon}(K) J_{\beta}(x K) \\
& =I(\mu, \epsilon, \eta, \beta)(x) .
\end{aligned}
$$

The Bessel reccurency law becomes reccurency law between Mellin integrals which can be written as:

$$
\begin{aligned}
I(\mu, \epsilon-1, \eta, \beta)= & 2(\mu+\epsilon-1) I(\mu, \epsilon, \eta+1, \beta)- \\
& I(\mu, \epsilon-2, \eta, \beta) .
\end{aligned}
$$

Now, we can use the Mellin transform tables to solve $I(x, \mu, \beta, \eta)$ and $I(x, \mu, \beta, \eta)$ which can be expressed as Mellin-Barnes integrals of the following type:

$\frac{1}{2 i \pi} \int_{c-i \infty}^{c+i \infty} G(t) F(1-t-\eta) x^{t+\eta-1} \mathrm{~d} t$

where $G$ and $F$ are the Mellin transform of $g$ and $f$ respectively. $g$ and $f$ are defined as:

$$
\begin{aligned}
& \left\{\begin{aligned}
f(a K) & =J_{\beta}(K x) \\
g(K) & =J_{\mu}^{2}(K)
\end{aligned} \quad \text { to solve: } I_{1}(x, \mu, \beta, \eta)(\mathrm{A} 15)\right. \\
& \left\{\begin{aligned}
f(a K) & =J_{\beta}(K x) \\
g(K) & =J_{\mu}(K) J_{\mu+1}(K)
\end{aligned} \quad \text { to solve } I_{2}(x, \mu, \beta, \eta) .(\mathrm{A} 16)\right.
\end{aligned}
$$

We have the Mellin transforms:

$$
\begin{aligned}
g(K)= & J_{\mu}^{2}(K) \longrightarrow G(s)=\frac{1}{2 \sqrt{\pi}} \Gamma\left[\begin{array}{c}
\frac{s}{2}+\mu, \frac{1-s}{2} \\
\mu+1-\frac{s}{2}, 1-\frac{s}{2}
\end{array}\right] \\
& \text { with } \operatorname{Re}(-2 \mu)<\operatorname{Re}(s)<1 \\
g(K)= & J_{\mu}(K) J_{\mu+1}(K) \longrightarrow G(s) \\
= & \frac{1}{2 \sqrt{\pi}} \Gamma\left[\begin{array}{c}
\frac{s+1}{2}+\mu, 1-\frac{s}{2} \\
\mu+\frac{3-s}{2}, \frac{3-s}{2}
\end{array}\right] \\
& \text { with } \operatorname{Re}(-2 \mu-1)<\operatorname{Re}(s)<2
\end{aligned}
$$

The Mellin transform of $f(\mathrm{Kx})$ is given by Eq. (A2). Finally, using the Cauchy's formula, we find:

If $x \leq 2$

$$
\begin{aligned}
& I_{1}(x, \mu, \beta, \eta)=\sum_{p=0}^{\infty} \frac{1}{2 \sqrt{\pi}} \frac{(-1)^{p}}{p !}\left(\frac{x}{2}\right)^{2 p+\eta} \\
& \Gamma\left[\begin{array}{c}
\frac{1}{2}+\mu+p, \frac{\beta}{2}-p-\frac{\eta}{2} \\
-p+\mu+\frac{1}{2}, \frac{1}{2}+p, p+\frac{\beta}{2}+1+\frac{\eta}{2}
\end{array}\right] \\
& +\sum_{p=0}^{\infty} \frac{1}{2 \sqrt{\pi}} \frac{(-1)^{p}}{p !}\left(\frac{x}{2}\right)^{2 p+\beta} \\
& \Gamma\left[\begin{array}{c}
\frac{1-\eta+\beta}{2}+\mu+p,-p+\frac{\eta+\beta}{2} \\
\mu-p-\frac{\beta+\eta+1}{2}, \frac{1+\eta-\beta}{2}-p, p+\beta+1
\end{array}\right]
\end{aligned}
$$

$$
\begin{aligned}
& I_{2}(x, \mu, \beta, \eta)=\sum_{p=0}^{\infty} \frac{1}{2 \sqrt{\pi}} \frac{(-1)^{p}}{p !}\left(\frac{x}{2}\right)^{2 p+\eta+1} \\
& \Gamma \quad\left[\begin{array}{c}
\frac{3}{2}+\mu+p,-p-\frac{\eta+1-\beta}{2} \\
\mu-p+\frac{1}{2}, \frac{1}{2}-p, p+\frac{\eta+3+\beta}{2}
\end{array}\right] \\
& +\sum_{p=0}^{\infty} \frac{1}{2 \sqrt{\pi}} \frac{(-1)^{p}}{p !}\left(\frac{x}{2}\right)^{2 p+\beta} \\
& \Gamma\left[\begin{array}{c}
\frac{1-\eta+\beta}{2}+\beta+p,-p+\frac{\eta+1+\beta}{2} \\
\mu-p+1+\frac{\eta-\beta}{2}, 1+\frac{\eta-\beta}{2}-p, p+\beta+1
\end{array}\right]
\end{aligned}
$$




\section{and $x>2$}

$$
\begin{aligned}
& I_{1} \quad(x, \mu, \beta, \eta)=\sum_{p=0}^{\infty} \frac{1}{2 \sqrt{\pi}} \frac{(-1)^{p}}{p !}\left(\frac{x}{2}\right)^{-2 p-2 \mu+\eta-1} \\
& \Gamma \quad\left[\begin{array}{c}
\frac{1}{2}+\mu+p, p+\mu+\frac{1-\eta+\beta}{2} \\
p+2 \mu+1, p+\mu+1,-p-\mu+\frac{\eta+1+\beta}{2}
\end{array}\right] \\
& I_{2} \quad(x, \mu, \beta, \eta)=\sum_{p=0}^{\infty} \frac{1}{2 \sqrt{\pi}} \frac{(-1)^{p}}{p !}\left(\frac{x}{2}\right)^{-2 p-2 \mu+\eta-2} \\
& \Gamma \quad\left[\begin{array}{c}
\frac{3}{2}+\mu+p, p+\mu+1-\frac{x+\beta}{2} \\
p+2 \mu+2, p+\mu+2,-p+\mu+\frac{x+\beta}{2}
\end{array}\right] .
\end{aligned}
$$

\section{References}

Rayrole J., 1992, “Themis technical report” (private communication)

Molodij G., Rayrole J., Madec P.Y., Colson F., 1996, "Performances Analysis For T.H.E.M.I.S. Image Stabilizer Optical System: I" A\&A 118, 169-179

Noll R.J., 1976, "Zernike polynomials and atmospheric turbulence", J. Opt. Soc. Am. A 66, 207-211

Kupke R., Roddier F., Mickey D.L., 1994, "Curvature-based wavefront sensor for use on extended patterns", SPIE Vol. 2201, 519-527

Rousset G., 1993, "Wavefront sensing" in Adaptive Optics For Astronomy, Alloin D., Mariotti J.M. (eds.) NATO ASI Series. Kluwer Academic Publisher 1993, p. 115-137

Gendron E., Lna P., 1994, "Astronomical Adaptive Optics I: Modal Control Optimisation", A\&A 291, 337-347

Chassat F., 1989, "Calcul du domaine d'isoplanètisme d'un système d'optique adaptative fonctionnant à travers la turbulence atmosphérique " J. Optics. (Paris) 20, 13-23

Sasiela R.J., 1994, "Electromagnetic Wave Propagation in Turbulence". Springer-Verlag

Molodij G., Rousset G., 1997, "Angular correlation of Zernike polynomials for a laser guide star in adaptive optics", J. Opt. Soc. Am. A

Primot J., Rousset G., Fontanella J.C. "Deconvolution from wavefront sensing: a new technique for compensating turbulence degraded image", J. Opt. Soc. Am 7, 1598

Chassat F., 1992, Thesis I: Propagation optique à travers la turbulence atmospherique. Étude modale de l'anisoplanetisme et application a l'optique adaptative

Barletti R., Ceppatelli G., Moroder E., Paterno L., Righini A., 1973, Site testing at Tenerife by ballon borne radiosonder and optical quality of the atlantic air mass over the Canary Island, J.O.S.O annual report

Wang J.Y., Markey J.K., 1978, "Modal compensation of atmospheric turbulence phase distortion", J. Opt. Soc. Am. A $68,78-87$

Valley G.C., Wandzura S.M., 1979, "Spatial correlation of phase-expansion coefficients for propagation through atmospheric turbulence" J. Opt. Soc. Am. A, 69, 712-717

Tyler G.A., 1990 "Analysis of propagation through turbulence: evaluation of an integral involving the product of three Bessel functions", J. Opt. Soc. Am. A. 7, 1218-1223

Roddier F., 1981, "The effect of atmospheric turbulence in optical astronomy", in Progress in Optics, Wolf E. (ed.). North-Holland publishing Co, Amsterdam, p. 19

Perrier C., 1989, "Amplitude estimation from speckle interferometry" in Diffraction Limited Imaging with very large Telescopes. Kluwer Academic Publishers, p. 99-111

Abitbolt M., Ben-Yosef N., 1991, "Partial correction estimation of adaptive optics systems for large fields of view", Waves in random Media, p. 99-112 